\title{
HERRAMIENTAS DE GAMIFICACIÓN PARA EL APRENDIZAJE DE CIENCIAS DE LA TIERRA
}

\section{GAMIFICATION TOOLS FOR LEARNING EARTH SCIENCES}

\author{
Isabel Sonsoles de Soto García \\ isabelsonsoles.desoto@unavarra.es \\ Universidad Pública de Navarra
}

\section{RESUMEN}

El porcentaje de abandono escolar en España es superior al de otros países de la Unión Europea, lo que hace necesario fomentar el rendimiento del alumnado y su motivación. En este contexto, la gamificación tiene un futuro prometedor ya que promueven la participación del alumnado y su deseo por aprender. Se han desarrollado tres actividades de gamificación (trivial, Kahoot y Socrative) para el estudio de ciencias de la tierra. Se ha observado que los alumnos prefieren las actividades de gamificación con soporte informático ya que crean un ambiente divertido que fomenta la participación y motivación. Sin embrago, también generan un aumento en la competitividad y los resultados obtenidos no son tan altos como los esperados inicialmente.

PALABRAS CLAVE: gamificación, enseñanza de ciencias de la tierra, niveles no universitarios, estrategias de motivación

\section{ABSTRACT}

The percentage of school dropouts in Spain is higher than in other countries of the European Union, which makes it necessary to encourage student school marks and motivation. In this context, gamification has a promising future since it promotes the participation of students and their desire to learn. Three gamification activities (trivial, Kahoot! and Socrative) have been developed for the study of Earth Sciences. It has been observed that students prefer gamification activities with computer support since these activities create a fun environment that encourages participation and motivation. However, they also generate an increase in competitiveness and the results obtained are not as high as initially were expected.

KEY WORDS: gamification, earth science education, non-university levels, motivation strategies 


\section{INTRODUCCIÓN}

El porcentaje de abandono escolar en el sistema educativo español es mayor que en otros países de la Unión Europea (UE). En el año 2009, el porcentaje de los jóvenes entre 18 y 24 años que abandonaron el sistema educativo fue del $31 \%$, frente al promedio europeo del $14.4 \%$. Además, los niveles de rendimiento son modestos si se comparan con los resultados obtenidos en otros países de la UE (Ayuste et al., 2012). Esto hace que sea necesario un cambio metodológico en la enseñanza y la necesidad de aplicar nuevas herramientas educativas que mejoren el rendimiento del alumnado, es decir, es necesario que el docente utilice herramientas innovadoras y nuevas estrategias didácticas que ayuden a fomentar la participación del alumnado y aumente su motivación. Ante esta necesidad, no se puede negar el enorme impacto de las tecnologías de la información y la comunicación (TICs) en los diferentes contextos sociales (Martínez Clares et al., 2016) y en especial en los centros educativos no universitarios, en donde la progresiva dotación de aulas digitales está generando paulatinamente un cambio metodológico, que ha permitido que las metodologías de aprendizaje colaborativo a través de las TICs presenten una importante proyección en procesos de innovación educativa (Hernández Martín y Martín de Arriba, 2017). Según Fernández-Miravete (2018), los estudiantes de educación secundaria que realizaron sus estudios dentro de un proyecto educativo TIC durante cuatro cursos académicos son más competentes digitalmente que aquellos estudiantes de modalidad tradicional. Sin embrago, en un estudio reciente sobre las TICs en 80 centros educativos madrileños, se observó que el buen uso de estas herramientas depende de varios factores como la formación y el perfil del profesorado, los recursos tecnológicos y pedagógicos disponibles, el uso que se le dan por parte del profesorado y de los alumnos, el equipo de coordinación tecnológica, etc. (Fernández Cruz et al., 2018).

En este contexto, las herramientas de gamificación parecen tener un futuro prometedor, ya que en la actualidad están surgiendo muchas aplicaciones informáticas con fines docentes en las que se utilizan videojuegos para adquirir todo tipo de contenidos. El término de gamificación, fue definido por Zichermann y Cunningham (2011), como "un proceso relacionado con el pensamiento del jugador y las técnicas de juego para atraer a los usuarios y resolver problemas", es decir, son actividades que permiten enseñar y reforzar conocimientos, además de adquirir habilidades como la resolución de problemas, la colaboración o la comunicación (Contreras Espinosa y Eguia, 2016).

Estas estrategias consisten en actividades en el aula en la que se utilizan juegos que permiten integrar clases dinámicas para aumentar la participación de los estudiantes en clases motivantes y conseguir que el estudiante "quiera aprender", es decir, potencian un aprendizaje más significativo (Kapp, 2012, Herberth Alexander, 2016, Molina Álvarez el al., 2017, Corchuelo-Rodriguez, 2018). Los videojuegos cautivan a los jóvenes, por lo que con esta herramienta innovadora que pretende llamar la atención de los alumnos, se puede fomentar su participación en el aula y motivar a los estudiantes. Además, permiten evaluar las actividades de forma continua e inmediata. 
Cuando una persona se divierte realizando una actividad, la nueva información se fija en el cerebro, por lo que utilizando este tipo de herramientas, se produce un aprendizaje óptimo y por tanto, un mejor aprendizaje (Molina Álvarez et al., 2017). En los juegos, el reto expresa la necesidad del jugador de conseguir superar sus expectativas, tiene una importante carga psicológica, cuyo principal fin es influir en el comportamiento del usuario (Díaz Cruzado y Troyano Rodríguez, 2013). Sin embargo, para obtener estos resultados en las aulas, se deben tener en cuenta varios aspectos. Según Herberth Alexander, 2016, las actividades de gamificación adaptadas a educación deben tener una estructuración compuesta por dinámicas centradas en retos, recompensas, logros, etc. con la finalidad, de hacer clases más atractivas para el alumnado y que además, mejorar sus resultados académicos al llevar a cabo una participación activa de la clase.

A pesar de que esta estrategia de educación aparece a principios de la década de los noventa del siglo XX, no ha sido muy utilizada en las aulas hasta hace pocos años. Este hecho queda demostrado al estudiar el número de artículos relacionados con herramientas de juego en el aula, ya que no existen trabajos de investigación relacionados con esta temática hasta una década después de su aparición y la mayor parte de estos artículos son de los últimos siete años (Martínez Jurado y Moyano Fuentes, 2017), por lo tanto, es un campo educativo nuevo pero con un rápido crecimiento. En la actualidad es una herramienta que se está utilizando en todos los niveles educativos desde primaria hasta niveles superiores como la universidad e incluso se ha utilizado en plataformas educativas (Benítez-Porres, 2015; ContrerasCastillo et al., 2015; Herberth Alexander, 2016, Quintaral Pérez, 2016, Molina Álvarez et al., 2017, Rodríguez-Fernández, 2017, Corchuelo-Rodriguez, 2018; Pérez Quiñones, 2018). Sin embargo, al ser una metodología tan novedosa, es necesario una mayor investigación para conocer más a fondo las ventajas e inconvenientes de dicha herramienta educativa. Por otro lado, respecto al uso de estas herramientas en el estudio de Ciencias de la Tierra, en la actualidad no existen materiales disponibles con fines docentes. Únicamente, se ha creado el primer videojuego educativo relacionado con esta materia (Earth Primer). Con esta aplicación, su creador Chaim Gingold, propone enseñar cómo trabajan las fuerzas geológicas de nuestro planeta a través del juego. Bajo este contexto, es necesario crear nuevos materiales relacionados con esta materia que permitan a los docentes trabajar en el aula de una forma más lúdica que facilite la motivación de su alumnado.

Las actividades de gamificaión se pueden dividir en dos grandes grupos. Por un lado, los juegos educativos convencionales que se caracterizan por no necesitar un soporte electrónico, como pueden ser las actividades de trivial y por otro lado, las actividades con soporte digital, siendo en la actualidad Kahoot! y Socrative las más utilizadas en las aulas. La principal diferencia que existe entre ambas metodologías es que la segunda muestra un espacio de juego más atractivo (Díaz Cruzado y Troyano Rodríguez, 2013), al incluir imágenes, sonidos, etc. lo que motiva más al alumnado.

Como se ha explicado anteriormente, los juegos educativos convencionales son actividades lúdicas que se realizan en el aula sin necesidad de un soporte electrónico con la finalidad de estimular el interés del alumnado y desarrollar actitudes positivas hacia las distintas materias. Son actividades ampliamente utilizadas en las aulas desde hace muchos años, en especial en niveles inferiores como primaria. Existen dos tipos 
de juegos: los que exigen a los jugadores que utilicen los conceptos vistos en el aula y los que exigen poner en práctica habilidades, razonamientos o destrezas (Gairin Sallan, 1990). Son actividades amenas que tienen gran utilidad en el aula pero a diferencia con las actividades de gamificación con soporte informático no facilitan un feedback inmediato.

La plataforma "Kahoot!" (https://kahoot.com/welcomeback/) es una herramienta educativa que puede ser utilizada por los alumnos y docentes desde un ordenador, tablet o un móvil con conexión a internet. Permite realizar rompecabezas, gráficos, discusiones y cuestionarios por lo que el profesorado cuenta con diferentes opciones para reforzar el aprendizaje de sus alumnos (Dellos, 2015, Pérez Quiñones, 2018). Es una herramienta muy fácil de utilizar donde los estudiantes no necesitan tener una cuenta Kahoot! y solo necesitan un dispositivo electrónico para acceder a los cuestionarios. Las preguntas se muestran en la pantalla del ordenador con distintos colores, junto con una melodía que recuerda a los videojuegos por lo que es un formato muy atractivo para el alumnado. Por otro lado, al finalizar la actividad, Kahoot! facilita los resultados obtenidos en la clase, por lo que de forma inmediata tanto el alumno como el profesor pueden ver cuáles han sido los aciertos y errores cometidos. Este feedback es útil y necesario en los procesos de aprendizaje y Kahoot! permite que este feedback sea bien recibido por los alumnos porque no lo aprecian como una crítica a su trabajo al estar en un ambiente lúdico.

Por otro lado, Socrative (https://www.socrative.com/) es una herramienta educativa que permite realizar actividades con preguntas de verdadero/falso, preguntas cortas y/o test de opción múltiple. La diferencia con Kahoot! es que el docente debe crear un aula virtual para que accedan sus alumnos y en dicha aula se encuentran disponibles las actividades, por lo que no es posible acceder a las actividades si el docente no ha facilitado el nombre de la clase virtual. Por otro lado, al igual que Kahoot!, este programa proporciona un feedback al alumnado mientras realiza la actividad y facilita al profesor un documento con las respuestas dadas por los alumnos en tiempo real.

\section{MATERIALES Y MÉTODOS}

Se han llevado a cabo tres actividades de gamificación (una actividad tipo trivial y dos actividades con soporte informático (Kahoot! y Socrative)) en un curso de Formación Profesional (FP) de grado medio para el aprendizaje de asignaturas relacionadas con Ciencias de la Tierra. En esta actividad se incluyeron a todos los alumnos del curso académico 2017-2018, concretamente una muestra de 15 alumnos. Estas actividades se realizaron en el aula en grupos de dos a tres alumnos. Posteriormente, se facilitó al alumnado la actividad para que tuvieran la posibilidad de repetir la actividad en casa de forma individual. Además, se utilizaron algunas de las preguntas de estas actividades en el examen de la unidad didáctica.

La actividad de trivial consiste en un tipo test sobre materiales terrestres, en la que los alumnos van contestado a unas preguntas en grupo y van ganando o perdiendo puntos en función del número de preguntas que contesten correctamente. Esta actividad se realizó durante una sesión de clase, el profesor formula una pregunta a un grupo y el resto de los grupos observan como contesta el equipo rival. En el caso de contestar 
adecuadamente a la pregunta, ese grupo suma cinco puntos y si la respuesta es incorrecta, resta dos puntos y la misma pregunta se formula al siguiente grupo de clase. Al finalizar la actividad, se hace un recuento de puntos y se observa que equipo ha conseguido mayor puntuación.

La actividad de Kahoot!, es también un tipo test, en este caso los contenidos están relacionados con minería. Esta actividad también se realizó en clase en grupos de dos a tres personas. En este caso, todos los alumnos contestan a la vez a la misma pregunta desde el ordenador de clase o desde sus dispositivos móviles. El resultado de las preguntas aparece en tiempo real en la pantalla del ordenador del profesor y todos los alumnos van viendo si su respuesta y la del resto de los equipos es correcta o incorrecta. Al final de la actividad, el programa facilita los resultados de los alumnos y muestra estos resultados en un podio, por lo que los alumnos pueden ver cuáles han sido los tres equipos con mejor puntación.

Por último, la actividad planteada con Socrative es una "Space Race" (carrera del espacio) sobre captaciones de agua subterráneas. En este tipo de actividades el alumnado compite por equipos por contestar correctamente a las actividades planteadas por el profesor. El objetivo de la actividad es ser la nave espacial más rápida de la clase.

Una vez terminadas las actividades se realizó un cuestionario de evaluación al alumnado sobre las herramientas de gamificación, en el que se pedía a los estudiantes que bajo su punto de vista valoraran las herramientas y señalaran con qué actividad consideraban ellos que habían aprendido más contenidos. La plataforma utilizada para este cuestionario fue "Encuestas de google".

\section{RESULTADOS Y DISCUSIÓN}

En las figuras 1 y 2 se presentan los resultados de la encuesta realizada a los alumnos sobre sus preferencias en cuanto a las herramientas de gamificación utilizadas en el aula. Como se puede observar, el alumnado prefiere actividades en las que tengan que utilizar las nuevas tecnologías, ya que la actividad del trivial no ha obtenido ningún voto. Además, consideran que su aprendizaje ha sido menor (Figura 1). Al igual que Benítez-Porres, 2015 en su estudio con Socrative en un grado universitario, las encuestas realizadas al alumnado sobre estas herramientas muestran una valoración muy positiva de las actividades de gamificación en las que se ha utilizado un soporte informático.

Los alumnos consideran que su aprendizaje es mayor cuando utilizan herramientas de gamificación con soporte electrónico, ya que el $60 \%$ del alumnado considera que ha aprendido más utilizando la herramienta Kahoot!, seguido del $40 \%$ de la clase que considera de mayor utilidad la herramienta Socrative. Sin embrago, ningún alumno ha votado a la actividad del trivial, actividad de gamificación convencional, realizada sin soporte tecnológico (Figura 1). 


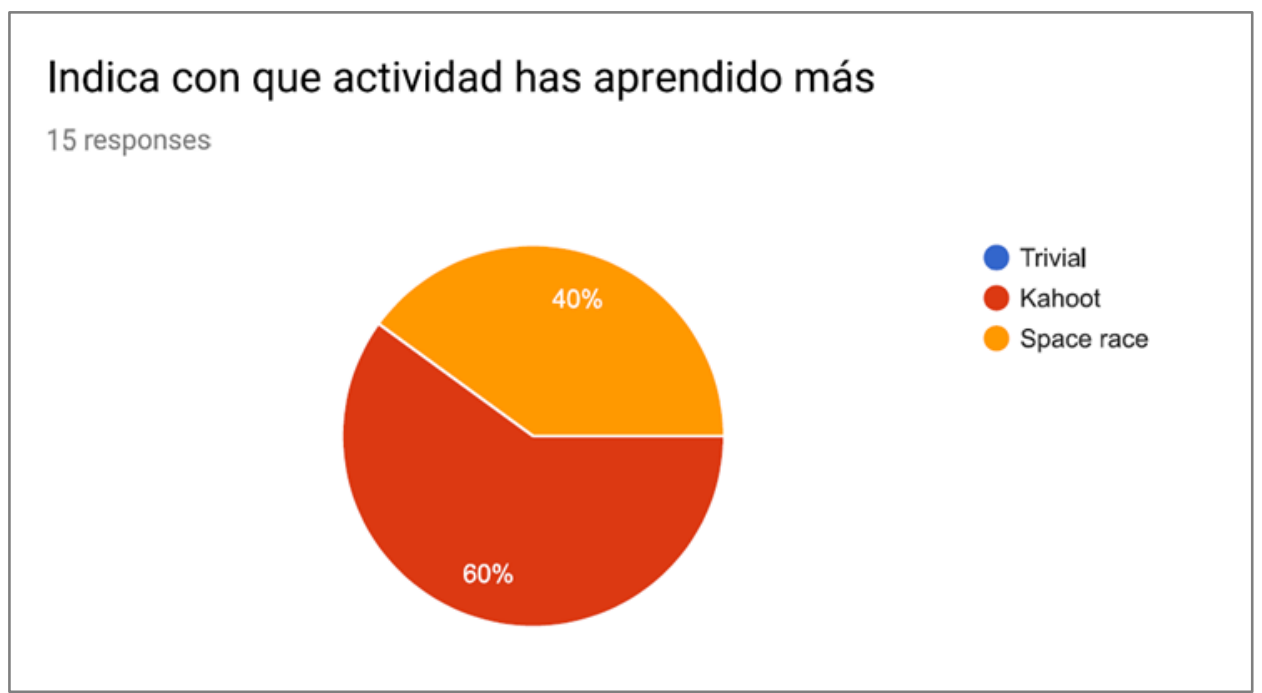

Figura 1: Resultado de la encuesta realizada a los alumnos sobre las herramientas de gamificación en la que se pregunta a los alumnos con qué actividad consideran ellos que han aprendido más.

Valora del 1 al 5 la actividad del trivial de rocas

15 responses

A

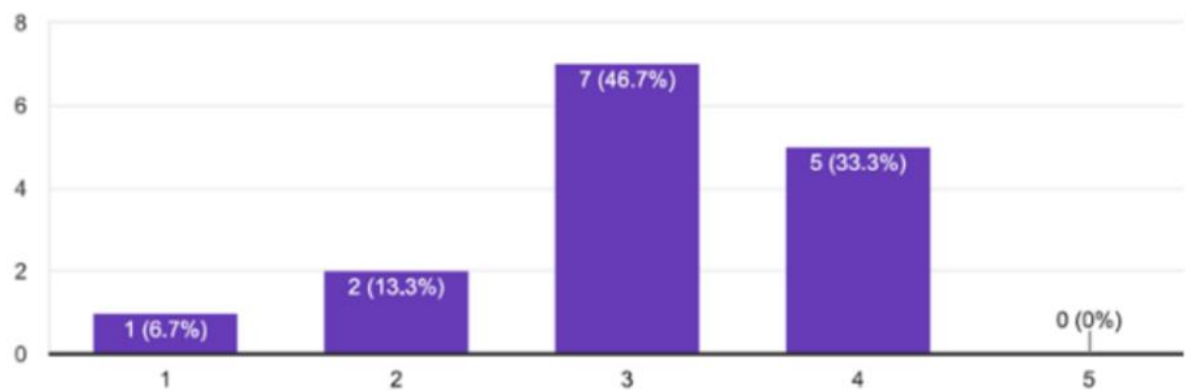

Figura 2: Resultado de la encuesta realizada a los alumnos sobre las herramientas de gamificación en la que se pide a los alumnos que valoren del uno al cinco las actividades realizadas. A: resultados de la actividad Trivial. 


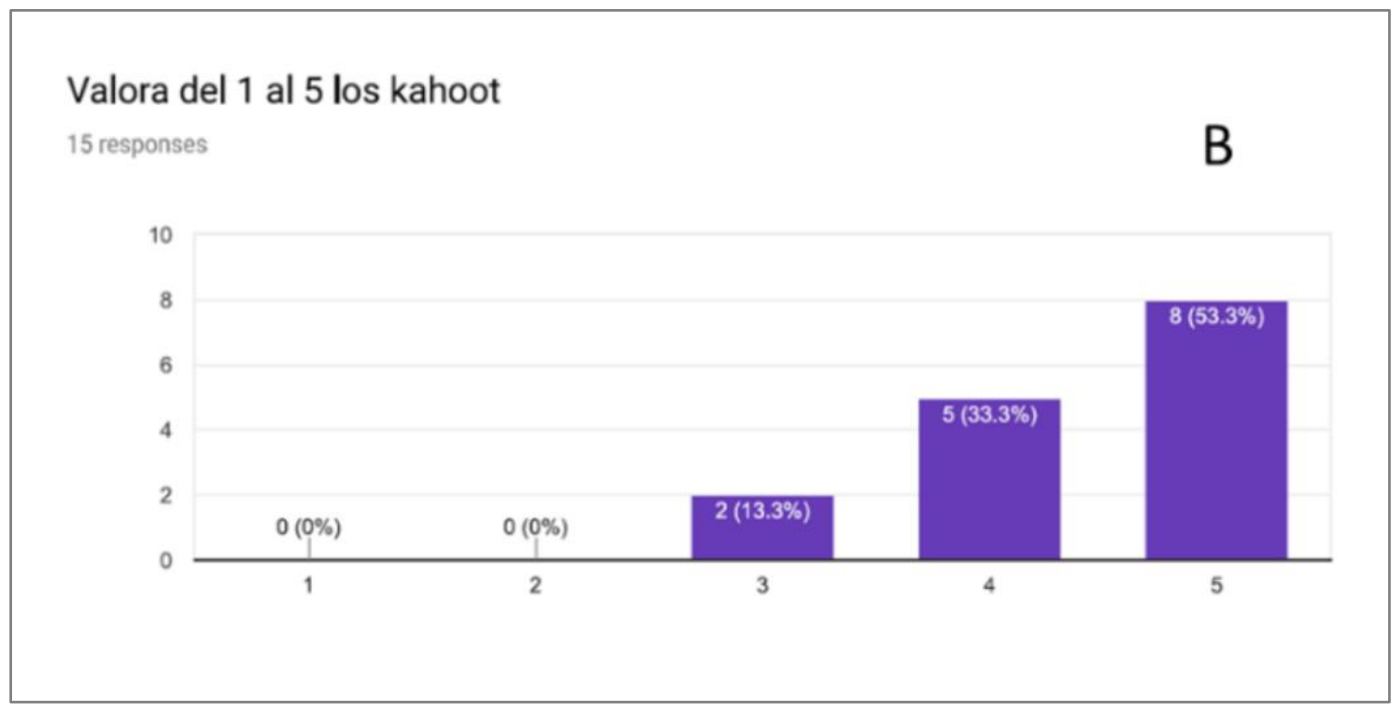

Figura 3: Resultado de la encuesta realizada a los alumnos sobre las herramientas de gamificación en la que se pide a los alumnos que valoren del uno al cinco las actividades realizadas. B: Resultados de la actividad Kahoot!.

Valora del 1 al 5 la "space race" de socrative 15 responses
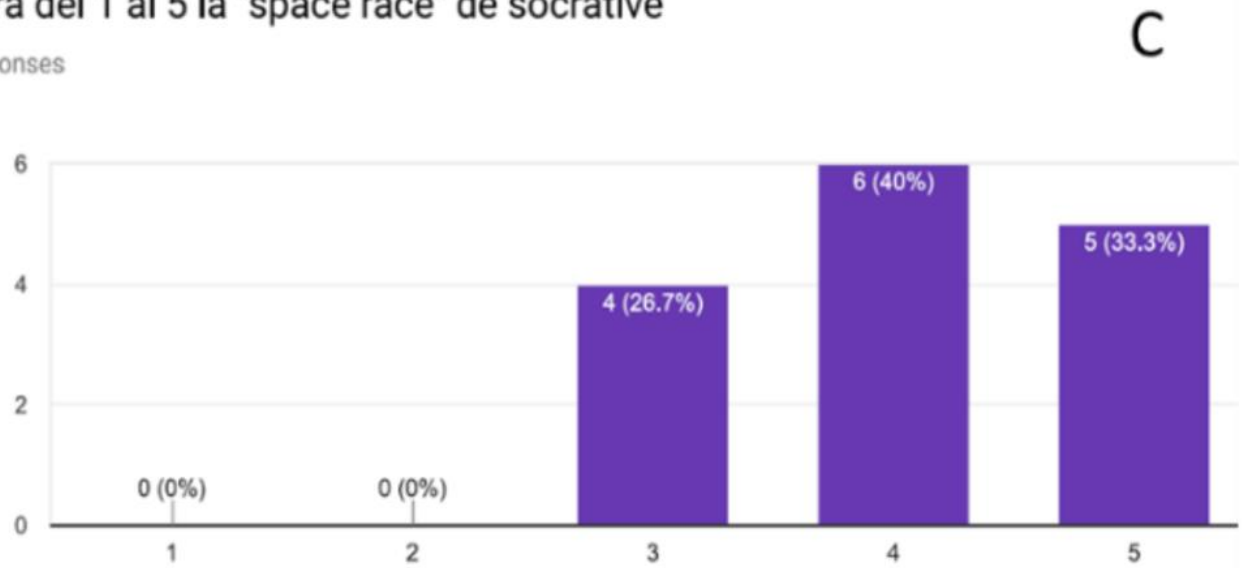

Figura 4: Resultado de la encuesta realizada a los alumnos sobre las herramientas de gamificación en la que se pide a los alumnos que valoren del uno al cinco las actividades realizadas. C: resultado de la actividad Socrative

Respecto a la puntuación de cada herramienta obtenida mediante una encuesta al alumnado (Figura 2), se observa que la actividad mejor valorada es el Kahoot! con 4.4 puntos, seguido de Socrative con 4 puntos y por último, la actividad del trivial con 3 puntos, lo que concuerda con los resultados de la Figura 1, donde se mostraba que el alumnado prefiere las actividades de gamificación innovadoras en las que se utilizan soportes informáticos. Por tanto, las herramientas de gamificación con soporte informático son altamente valoradas por el alumnado, ya que lo perciben como una herramienta que promueve el aprendizaje de los contenidos vistos en clase. Estos datos concuerdan con los obtenidos recientemente por García-Valcárcel y Tejedor Tejedor, (2017) en un estudio sobre el rendimiento académico con el uso de las TICs, 
observaron que los alumnos de mayor éxito académico reconocían en las TICs un mayor potencial de apoyo en sus estrategias de aprendizaje.

Los resultados académicos de los estudiantes (datos no presentados) han mostrado que se observa un mayor interés del alumnado por la asignatura con el uso de esta técnica ya que se logra despertar en el estudiante la necesidad de aprender al crear un ambiente divertido y competitivo en el aula. Además, gracias al feedback que proporciona esta técnica, tanto el estudiante como el docente conocen de forma inmediata los progresos realizados, es decir, existe una retroalimentación constante. Estas resultados concuerdan con los obtenidos por Herberth Alexander, 2016 en un contexto universitario. Por otro lado, al facilitar al alumnado las actividades de gamificación para que puedan realizar en casa de nuevo la actividad, se observó que la repetición de la actividad contribuye directamente en la efectividad, mejorando los resultados de los exámenes al compararlo con los resultados de años anteriores.

Sin embargo, al igual que Hamari et al., (2014), Borras Gené, (2015) y Hanus y Fox, (2015), se ha observado que la gamificación presenta puntos negativos como son el aumento de la competitividad entre el alumnado, la desmotivación de los alumnos que se encuentran en puestos inferiores del ranking y que los resultados obtenidos son más bajos que los esperados ya que el alumnado se aprende de memoria las preguntas sin relacionar contenidos. Por esta razón, las herramientas de gamificación son muy útiles para conocer los conocimientos previos sobre la temática de la sesión o bien afianzar contenidos ya vistos a lo largo de una unidad didáctica (Benítez-Porres, 2015), pero no como herramienta de evaluación del alumnado. Estas actividades se deben realizar de forma ordenada de tal forma que cubra las necesidades de cada grupo de alumnos (Molina Álvarez el al., 2017) y de ese modo se eviten estos puntos negativos.

\section{CONCLUSIONES}

Tras la realización de este estudio se puede llegar a las siguientes conclusiones:

- Las herramientas de gamificación crean un ambiente divertido en las aulas que fomentan la participación del alumnado y la motivación del mismo.

- Son herramientas útiles como actividades de iniciación o para afianzar los contenidos en clase pero no son útiles como herramientas de evaluación.

- El alumnado prefiere las herramientas de gamificación con soporte informático como Kahoot! o Socrative ya que considera que su aprendizaje es mayor.

- Los inconvenientes observados de la gamificación es que aumenta la competitividad del alumnado y que los resultados obtenidos son más bajos que los esperados, ya que el alumno se aprende las preguntas de memoria. 


\section{REFERENCIAS}

Ayuste, A., Gros, B. \& Valdivieso, S. (2012). Sociedad del conocimiento. Perspectiva pedagógica. En García Aretio, L., (Ed.) Sociedad del Conocimiento y Educación (pp. 17-40). Editorial UNED, Madrid (España).

Benítez-Porres, J. (2015). Socrative como herramienta para la integración de contenidos en la asignatura "Didáctica de los Deportes". XII Jornadas Internacionales de Innovación Universitaria, Villaviciosa de Odón, 20 y 21 de julio, 2015. Recuperado de: http://abacus.universidadeuropea.es/handle/11268/4513

Borrás Gene, O. (2015). Fundamentos de la gamificación. Gabinete de Tele-Educación. Universidad Politécnica de Madrid. Recuperado de: http://oa.upm.es/35517/1/fundamentos\%20de\%20la\%20gamificacion v1 1.pdf

Contreras-Castillo J., Baron-Ramirez N., Acosta-Díaz R., Guerrero-lbañez A., FigueroaPerez J., \& Arce-Garcia A. (2015). Gamificación en Plataformas Educativas. Memorias del XXI Congreso Internacional sobre Educación Bimodal. Medellín Colombia. 25-27 noviembre 2015. Pags. 16-31

Contreras Espinosa, R. S. \& Eguia, J. L. (2016): Gamificación en aulas universitarias. Bellaterra: Institut de la Comunicació, Universitat Autònoma de Barcelona. Recuperado de: http://incom.uab.cat/download/eBook_incomuab_gamificacion.pdf

Corchuelo-Rodriguez C. A. (2018). Gamificación en la educación superior: Experiencia innovadora para motivar estudiantes y dinamizar contenidos en el aula. Revista Electrónica de Tecnología Educativa 63, 29-41. Recuperado de: http://www.edutec.es/revista/index.php/edutec-e/article/view/927

Dellos, R. (2015). Kahoot! A digital game resource for learning. International Journal of Instructional Technology and Distance Learning 12(4): 49-52. Recuperado de: http://www.itdl.org/Journal/apr_15/apr15.pdf

Díaz Cruzado, J. \&Troyano Rodríguez, Y. (2013). El potencial de la gamificación aplicado al ámbito educativo. III Jornadas de Innovación Docente. Innovación Educativa: respuesta en tiempos de incertidumbre. Recuperado de: https://idus.us.es/xmlui/handle/11441/59067

Pérez Quiñones, F. C. (2018) Aplicación móvil Kahoot! para la práctica del aprendizaje en los estudiantes de educación media superior. En: Barraza Macías, A. (Ed). Siete proyectos de innovación educativa, Más allá de la didáctica. Méjico 9-18 $\mathrm{pp}$

Fernández Cruz, F.J.; Fernández Díaz, M.J. \& Rodríguez Mantilla, J.M. (2018). EI proceso de integración y uso pedagógico de las TIC en los centros educativos madrileños. Educación XX1 21(2): 395-416. Recuperado de: http://revistas.uned.es/index.php/educacionXX1/article/view/17907 
Fernández-Miravete A. D. (2018). La competencia digital del alumnado de Educación Secundaria en el Marco de un proyecto educativo TIC (1:1). Revista Electrónica de Tecnología Educativa 63, 60-72. Recuperado de: http://www.edutec.es/revista/index.php/edutec-e/article/view/1027

Gairín Sallán, J. M. (1990). Efectos de la utilización de juegos educativos en la enseñanza de las matemáticas. Educar 17:105-118. Recuperado de: http://educar.uab.cat/article/view/v17-gairin

García-Valcárcel, A. \& Tejedor Tejedor, F. J. (2017). Percepción de los estudiantes sobre el valor de las TIC en sus estrategias de aprendizaje y su relación con el rendimiento. Educación XX1, 20(2), 137-159. Recuperado de: http://revistas.uned.es/index.php/educacionXX1/article/view/19035

Hamari, J., Koivisto, J. \& Sarsa, H. (2014). Does gamification work? A literature review of empirical studies on gamification. $7^{\text {th }}$ International Conference on System Science. Hawaii. Recuperado de: https://people.uta.fi/ kljuham/2014hamari_et_al-does_gamification_work.pdf

Hanus, M.D. \& Fox, J. (2015). Assessing the effects of gamification in the classroom: A longitudinal study on intrinsic motivation, social comparison, satisfaction, effort, and academic performance. Computers \& Education, 80, 152-161. Recuperado de: https://www.sciencedirect.com/science/article/pii/S0360131514002000

Herberth Alexander, O. (2016) La gamificación como estrategia metodológica en el contexto educativo universitario. Realidad y Reflexión, $\mathrm{N}^{\circ}$ 44: 29-47. Recuperado de: https://www.lamjol.info/index.php/RyR/article/view/3563

Hernández Martín, A. \& Martín de Arriba, J. (2017). Concepciones de los docentes no universitarios sobre el aprendizaje colaborativo con TIC. Educación XX1, 20(1), 185-208.

Recuperado

de:

http://revistas.uned.es/index.php/educacionXX1/article/view/17508

Kapp, K, M. (2012). The gamification of learning and instruction: Game-based methods and strategies for training and education. San Francisco: Pfeiffer

Martínez Clares, P., Pérez Cusó, J. \& Martínez Juárez, M. (2016). Las TICS y el entorno virtual para la tutoría universitaria. Educación XX1, 19(1), 287-310, doi:10.5944/educXX1.13942.

Recuperado

de:

http://revistas.uned.es/index.php/educacionXX1/article/view/13942

Martínez Jurado, P. J. \& Moyano Fuentes, J. (2017). Aprendiendo a Enseñar Lean Management mediante Juegos: Revisión Sistemática de la Literatura. Working Papers on Operations Management 8: 164-170

Molina Álvarez, J.J., Ortiz Colón. A.M., \& Agreda Montoro, M. (2017). Análisis de la integración de procesos gamificados en Educación Primaria. En Ruiz Palmero, J., Sánchez-Rodríguez, J. \& Sánchez-Rivas, E. (Ed.). Innovación docente y uso de las TIC en educación. Málaga: UMA Editorial. Recuperado de: http://www.enriquesanchezrivas.es/congresotic/archivos/Form_Compet_metod os/Ortiz_Otros_2.pdf 
Quintanal Pérez, F. (2016). Aplicación de herramientas de gamificación en física y química de secundaria. Opción, vol. 32 (12): 327-348. Recuperado de: https://dialnet.unirioja.es/servlet/articulo?codigo $=5852309$

Rodríguez-Fernández, L. (2017). Smartphones y aprendizaje: el uso de Kahoot en el aula universitaria. Revista Mediterránea de Comunicación/Mediterranean Journal of Communication, 8(1), 181-190. Recuperado de: https://www.mediterranea-comunicacion.org/article/view/2017-v8-n1smartphones-y-aprendizaje-el-uso-de-kahoot-en-el-aula-universitaria

Zichermann, G. \& Cunningham, C. (2011). Gamification by Design: Implementing Game Mechanics in Web and Mobile Apps. Cambridge, MA: O'Reilly Media.

Para referenciar este artículo:

de Soto García, I. S. (2018). Herramientas de gamificación para el aprendizaje de ciencias de la tierra. Edutec. Revista Electrónica de Tecnología Educativa, 65, 29-39. doi:https://doi.org/10.21556/edutec.2018.65.1143 